See discussions, stats, and author profiles for this publication at: https://www.researchgate.net/publication/318983666

\title{
Framework of Synchromodal Transportation Problems
}

Conference Paper · October 2017

DOI: 10.1007/978-3-319-68496-3_26

CITATIONS

READS

155

5 authors, including:

Dylan Huizing

(3.) Centrum Wiskunde \& Informatica

3 PUBLICATIONS 6 CITATIONS

SEE PROFILE

Alex Sangers

TNO

14 PUBLICATIONS 12 CITATIONS

SEE PROFILE

Some of the authors of this publication are also working on these related projects:

Project Enhanced geometric models for access networks. View project

Predictive Synchromodality View project 


\title{
Framework of Synchromodal Transportation Problems
}

\author{
M.A.M. De Juncker ${ }^{13}$, D. Huizing ${ }^{23}$, M.R. Ortega del Vecchyo ${ }^{23}$, F. \\ Phillipson $^{3}$ and A. Sangers ${ }^{3}$ \\ 1 Eindhoven University of Technology, The Netherlands, \\ ${ }^{2}$ Delft University of Technology, The Netherlands, \\ 3 TNO, PO Box 96800, 2509 JE The Hague, The Netherlands
}

\begin{abstract}
Problem statements and solution methods in mathematical synchromodal transportation problems depend greatly on a set of model choices for which no rule of thumb exists. In this paper, a framework is introduced with which the model choices in synchromodal transportation problems can be classified, based on literature. This framework should help researchers and developers to find solution methodologies that are commonly used in their problem instance and to grasp characteristics of the models and cases in a compact way, enabling easy classification, comparison and insight in complexity. It is shown that this classification can help steer a modeller towards appropriate solution methods.
\end{abstract}

Keywords: Synchromodal, Classification, Logistics, Computation Models

\section{Introduction}

Synchromodal freight transport is a relatively new concept within the logistics sector. Older concepts of logistics are multimodal and intermodal. A transportation network is called a multimodal transport network if the transportation of goods can be made via different modes, where a mode is understood as a means of transportation, such as a barge. In an intermodal transportation network, the goods are transported through a standardised unit of transportation, which we call freight, and in practice is usually a container. Synchromodal freight transport is viewed in this paper as intermodal freight transport with an increased focus on at least one of the following two aspects:

1. Transport planning is done using real-time data, allowing for on-line changes in the planning; $[27,32,40,37]$

2. Different parties share their real-time information, transportation resources or transportation demands and may even entrust decisions to a central operator or logistics service provider (LSP). In some cases, clients may make an a-modal booking, agreeing with an LSP that their goods will be delivered at a set time and place against a set price and leaving it up to the LSP by what modes this is done. [27, 35, 51, 32, 40] 
Though other important developments exist within intermodal transport [47], synchromodality only concerns synchronising real-time data collection with realtime planning and synchronising the transportation flows and requirements among different parties. The goal of aspect 1 is to increase flexibility and reliability, that is to say, to become able to deal with disturbances in the system more effectively and to more effectively optimise against unknowns. The goal of aspect 2 is to increase efficiency and sustainability, by facilitating full truck load-consolidation (FTL-consolidation), in other words, letting one small order wait at a terminal so it can be combined with some other order [49]. Aspect 2 also facilitates smarter equipment repositioning, for example, by moving leftover empty containers directly to a nearby terminal where they are needed instead of through a depot [2].

Interest in synchromodality has increased, due to improvements in data technology, an increased focus on the more complicated hinterland transport and the ever-growing need for efficiency. However, synchromodality faces several challenges that keep it from being adopted in practice. The challenges come from several sources. In [32], seven critical success factors of synchromodality are discussed:

1. Network, collaboration and trust

2. Awareness and mental shift

3. Legal and political framework

4. Pricing/cost/service

5. ICT/ITS technologies

6. Sophisticated planning

7. Physical infrastructure

Roughly, it can be argued that the first and second factor are mainly social problems, the third is a political problem, the fourth is a mathematical, social and political problem, the fifth is a technological problem, the sixth is a mathematical problem, and the seventh is a technological and constructional problem.

Each of these factors is currently being addressed by different initiatives. Also in mathematics (applied in logistics) a lot of work has been done that can be used in synchromodality. Mathematical planning problems are often divided into three main categories: strategical, tactical, and operational, so is the case with mathematical synchromodal problems. These problems are related in a pyramidal-like structure in the following sense: tactical problems are usually engaged where a specific strategical instance is given, and operational problems are frequently solved where a strategical and tactical structure are fixed, although sometimes problems in two consecutive levels are solved simultaneously: for instance, in [8], the frequency of a resource is determined along with the flow of freight (that is, part of the schedules to resource and the freight to resources are solved at once). Mathematical synchromodal transportation problems on a tactical or operational level are usually represented via tools from graph theory and optimisation [39]. However, more often than not, the similarities end there: most of the models used to analyse a synchromodal transportation network are targeted to a specific real problem of interest [39], and knowledge and methods of other branches such as 
statistics, stochastic processes, or systems and control are often used. The models emphasise on what is most important for the given circumstances. Consequently, mathematical synchromodal transportation problems on a tactical or operational level have been engaged with approaches that may differ in many aspects:

- The exhaustiveness of the elements considered varies, e.g. weather or traffic conditions are considered in some models (such as the one presented in [23]) but not all.

- The elements that can be manipulated and controlled may vary, e.g. the departure time of some transportation means may be altered if suitable (as it happens in the model of [8]) or it may be that all transportation schedules are fixed.

- The amount of information relevant to the behaviour of the network may vary, and if a lack of information is considered, the way to model this situation may also vary [31].

- Whether some other stakeholders with authority in the network are in the model, and if so, how their behaviour is modelled.

A model is not necessarily improved by making it increasingly exhaustive. As it happens with most model-making, accuracy comes with a trade-off, in this case, computational power. This computational burden is an intrinsic property of operational synchromodal problems [48] and one that is of the utmost importance given the real-time nature of operational problems: new information is constantly fed and it should be processed on time.

There is no rule of thumb for making the decisions above; also, each of the decisions mentioned above will shape the model, and likely stir its solution methods to a specific direction. Though literature reviews of synchromodal transportation exist $[48,39]$, no generalised mathematical model for synchromodal transportation problems has been found yet, nor a way of categorising the existing literature by their modelling approaches. The framework for mathematical synchromodal transportation problems on a tactical or operational level presented in this paper aims to capture the essential model-making decisions done in the model built to represent the problem. When no such model is specified, it shows the model-making decisions likely to be done in that case, which makes classification partly subjective. This is done in an attempt to grasp the characteristics of the model/case in a compact way, enabling easy classification and comparison between models and cases, as well as a way to see the complexity of a specific case at a glance. Also, it provides perspective to better relate new problems with previous ones, thus identifying used methodologies for the problem at hand.

In the remainder of this paper, Section 2 gives an overview of the relevant literature. Section 3 introduces the classification framework and Section 4 two shorthand notations for this framework. In Section 5, some examples are provided. Based on these examples, common solution methods are mapped in Section 6 and the relationship with VRP terminology is discussed in Section 7. In Section 8 the examples are used to discuss strengths and weaknesses of the framework. 


\section{Literature}

Synchromodal planning problems exist both in the tactical and operational area. The tactical planning problem is quite extensive. One needs to select and schedule the services to operate, allocate the capacity and equipment, and look at the routing of the goods. Together this is also called Service Network Design. The review paper of Crainic [13] gives an extensive review of these problems, their formulations and their solution frameworks. They also give a classification of these problems. In the literature these problems are mostly modelled as FixedCost Capacitated Multicommodity Network Design Problems. The paper by Min [29] develops a chance-constrained goal programming model that has multiple aspects in the objective function.

Papers in this area that explicitly deal with synchromodality are $[36,11,8]$. The paper by Puettmann and Stadtler [36] mentions the importance of coordination of plans and operation of independent service providers in an intermodal transportation chain. They present a coordination scheme that will lead to reductions in overall transportation costs. They include stochastic demand in their calculation of the overall costs. Another paper by Caris, Macharis and Janssens [11] also looks at cooperation between inland terminals. In the paper they develop a service network design model for intermodal barge transport and apply it to the hinterland network of the port of Antwerp. They simulate cooperation schemes to attain economies of scale. The paper by Behdani et al. [8] develops a mathematical model for a synchromodal service schedule on a single origin-destination corridor. Taking into account the frequency and capacity of different modalities, it determines the optimal schedule and timing of services for all transport modes. The assignment of containers to services is also determined by the model.

In operational planning problems, problems are regarded that deal with the day-to-day problems in a logistic network. This means that all these problems deal with uncertainty and stochasticity, which makes these problems complex. The decisions depend on the current information and an estimation of the future events. Issues here are:

- reliability of a network: dealing with disruptions $[19,12,28,33]$ and resilience measures [12, 28];

- resource management: empty unit repositioning problems $[14,16,15]$ and allocation and positioning of the operating fleet [42-46, 7,38];

- replanning and online allocation $[10,17,21]$.

Papers in the operational area within the synchromodal context are [51,27,31]. Zhang and Pel [51] developed a model that captures relevant dynamics in freight transport demand and supply, flexible multimodal routing with transfers and transhipments. It consists of a demand generator (random sampling from historic data), an infrastructure and service network processor (which generates the resource schedule), a schedule-based assignment module (which assigns the demand to resources) and a performance evaluator. The model can be used to 
compare intermodal and synchromodal transportation from different perspectives: economic, social and environmental. The authors use their model for a case study regarding the Rotterdam hinterland container transport and they show that synchromodality will likely improve service level, capacity utilisation and modal shift, but not reduce delivery cost.

The paper by Mes and Iacob [27] searches for the $k$-shortest paths through an intermodal network. They present a synchromodal planning algorithm that takes into account time-windows, schedules for trains and barges and closing times of hubs and minimises costs, delays and $\mathrm{CO}_{2}$ emissions. The $k$-shortest paths are then presented to a human planner, which can choose the best fitting path for an order by filtering these paths. Their approach consists of offline steps and online steps. In the offline steps, the network is reduced by eliminating paths that are too far from the route. In the online steps an order is assigned to paths, by iterating over the number of main legs. A main leg in this paper is a certain train or barge. The assumption they make is that a cost efficient route consists of as few legs as possible. The online steps can be done after a disruption to make a new planning.

The paper by Rivera and Mes [31] looks at the problem of selecting services and transfers in a synchromodal network over a multi-period horizon. They take into account the fact that an order can be rerouted at any given moment. The orders become known gradually, but the planner has probabilistic knowledge about their arrival. The objective is to minimise expected costs over the entire horizon. They propose a Markov Decision Process model and a heuristic approach based on approximate dynamic programming.

\section{Framework identifiers and elements}

In this section the framework is introduced. Within the framework demand and resources are considered. In synchromodal transportation models, demand will likely be containers that need to be shipped from a certain origin to a destination. Resources can for example be: trucks, train and barges. However, the framework allows for a broader interpretation of these terms. In repositioning problems, empty containers can be regarded as resources, where the demand items are bulks of cargo that need to be put in a container.

The framework has two main parts. The first part consists of the identifiers; these are specific questions one can answer about the model that depict the general structure of the model. The other is a list of elements; these elements are used to depict in more detail what the nature is of the different entities of the synchromodal transportation problem. Note that the notation presented does not include the optimisation objective. Within a specific model there is of course an option to look at different optimisation objectives. This framework is developed in collaboration with multiple parties that study synchromodal systems. However, for certain specific problems one might want to extend the framework. We think this is easily done in the same way as we set up the framework. 


\subsection{Identifiers}

First we will elaborate on the identifiers of the framework. These identifiers are questions about the model. They identify the number of authorities, i.e. how many agents are in control of elements within the model. They will also identify the nature of different elements within the model. The list of elements will be discussed in detail in Section 3.2, but they are used to determine which components in the model are under control, which are fixed, which are dynamic and which are stochastic. For instance, the departure time of a barge may be a control element, but it could also be fixed upfront, or modelled as stochastic. Some of the questions address how the information is shared between different agents and if the optimisation objective is aimed at global optimisation or local optimisation. All the answers on these questions together present an overview of the model, which can then be easily interpreted by others or compared to models from the literature.

The identifiers that describe the behaviour of the model in more detail are discussed below. Note that 'resources' most often refer to transport vehicles and 'demand items' most often refer to freight containers: however, demand items could also be empty containers with no specific destination in equipment repositioning problems. Therefore, a degree of generality is necessary in these identifiers.

1. Are there other authorities (i.e. agents that make decisions)?

Here it is identified if there is one global controller that steers all agents in the network or that there are multiple agents that make decisions on their own.

- If there are other authorities, how is their behaviour modelled: One turn only, Equilibrium or Isolated?

If the previous question is answered with yes, i.e., there are multiple agents that make decisions, one needs to specify how these authorities react to each other. Three different ways for modelling the behaviour of multiple authorities in a synchromodal network are distinguished:

- One turn only: this means that each agent gets a turn to make a decision. After the decision is made, the agent will not switch again. For instance, in the case of three agents $A, B$ and $C$, agent $A$ will first make a decision, then agent $B$ and then agent $C$. The modelling ends here, since agent $A$ will not differ from its first decision.

- Equilibrium: the difference between "one turn only" and "equilibrium" is that after each agent has decided, agents can alter their decision with this new knowledge. In the same example: agents $A, B$ and $C$ make a decision, but then agent $A$ changes its decision based on the decisions of $\mathrm{B}$ and $\mathrm{C}$. If nobody wants to alter their decision anymore the modelling ends and an equilibrium is reached between the specific agents.

- Isolated: if the behaviour of the multiple authorities is isolated, it means that from the perspective of one of the authorities only limited 
information is available about the decisions of the other agents. For instance: agent $C$ needs to make a decision. It is not known what agents $A$ and $B$ have chosen or will choose, but agent $C$ knows historic data on the decisions of agents $A$ and $B$. Agent $C$ can then use this information to make an educated guess on the behaviour of agents $A$ and $B$.

2. Is information within the network: global or local?

This identifies if the information within the network is available globally or locally. If the information is locally available, it means that only the agents themselves know for example where they are or what their status is at a certain time. If the information is global, the network operator and/or all other agents know all this information as well.

3. Is the optimisation objective: global or local?

The same can hold for the optimisation objective. If all agents need to be individually optimised, the optimisation objective is local. If the optimisation objective is global, we want the best alternative for the entire network.

4. Which elements do you control?

Since we want to model a decision problem, at least one element of the system must be in control and must take decisions. For example: if one wants to model which containers will be transported by a certain mode in a synchromodal network, we have control over the demand-to-resource allocation. If we want to model which trains will depart on which time at certain locations, we have control of the resource departure time. An extensive list of elements is given in Section 3.2.

Of course the controllable element can have constraints: for instance, we can influence the departure times of trains, but they cannot depart before a certain time in the morning. This is still a controllable element. We thus consider an element a controllable element if a certain part of it can be controlled.

5. What is the nature of the other elements (fixed, dynamic, stochastic or irrelevant)?

The other elements within the network can also have different behaviour. We distinguish four:

- Fixed: a fixed element does not change within the scope of the problem.

- Dynamic: a dynamic element might change over time or due to a change in the state of the system (e.g. the amount of containers changes the travel time), but this change is known or computable beforehand.

- Stochastic: a stochastic element is not necessarily known beforehand. For instance, it is not known when orders will arrive, but it is a Poisson process. It might also occur that the time the order is placed is known, but the amount of containers for a certain order follows a normal distribution.

- Irrelevant: the list we propose in Section 3.2 is quite extensive. It might occur that for certain problems not all elements are taken into consideration to model the system. Then these elements are irrelevant. 


\section{What is the optimisation objective?}

This identifier is for the optimisation objective. One can look at the exact same system but still want to minimise a different function. One could think of travel times and $\mathrm{CO}_{2}$ emissions. It is also possible to identify a much more specific optimisation objective. Examples of optimisation objectives are in Section 5 .

\section{$3.2 \quad$ Elements}

Having defined the identifiers of the framework, now a list of elements is presented, that are expected to exist in most synchromodal transportation problems. They are divided in two parts: resource elements and demand elements. The resource elements are all elements related to the resources, which are mostly barges, trains and trucks. However, for compactness we also view a terminal as a resource. In the demand elements are all elements related to the demand, which are most of the time freight or empty containers. Most elements mentioned in this list are straightforward, small clarifications are mentioned where necessary.

- Resource elements:

- Resource Type: Different modalities can be modelled as different resource types. Another way to use this element is for owned and subcontracted resources.

- Resource Features: These features can be appointed to the different resource types or can have the same nature for the different types. For instance, it may be that there are barges and trains in the problem, but their schedules are both fixed, thus making the nature of the resource features fixed for both resource types.

* Resource Origin $(R O)$;

* Resource Destination (RD);

* Resource Capacity $(R C)$ : Indication of how much demand the different resources can handle;

* Resource Departure Time (RDT);

* Resource Travel Time (RTT): Time it takes to travel from the origin to the destination (in the case of a moving resource);

* Resource Price (RP): This can be per barge/train/truck/... or per container.

- Terminal Handling time (TH): Time it takes to handle the different types of modes at the terminal. This can again be per barge/train/truck/... or per container.

- Demand elements:

- Demand Type: One can also think of different types of demand. For instance, larger and smaller containers or bulk.

- Demand-to-Resource allocation (D2R): The assignment of the demand to the resources.

- Demand Features:

* Demand Origin $(D O)$; 
* Demand Destination $(D D)$;

* Demand Volume $(D V)$ : It might be that different customers have different amount of containers that is being transported. (Note that the demand element in this case will always be 1 container, since each container can have its own assignment.);

* Demand Release Date (DRD): The release date is the date at which the container is available for transportation;

* Demand Due Date $(D D D)$ : Latest date that the container should be at its destination, which is not necessarily a hard deadline;

* Demand Penalty (DP): Costs that are incurred when the due date is not met or when the container is transported before the release date (this is sometimes possible with coordination with the customers).

\section{Notation}

In this section, two types of notation are introduced, which will make it easier to quickly compare different models. Obviously, it is hard to make a compact notation and still incorporate all aspects of a synchromodal system. Therefore, the notation was made as compact as possible and some of the details are left out. When comparing models in detail, it is easier to look at all answers to the identifiers mentioned in Section 3.1. Our six-field notation was built to resemble Kendall's notation for classification of queue types [20] and the notation of theoretic scheduling problems proposed by Graham, Lawler, Lenstra and Rinnooy Kan [18].

\subsection{Six-field notation}

A synchromodal transportation model can be described by the notation:

$$
C|S| D|I| Y \mid B
$$

The letters denote the following things:

$-C$ : controlled elements,

$-S$ : stochastic elements,

- D: dynamic elements,

- I: irrelevant elements,

- $Y$ : system characteristics,

$-B$ : behaviour of other authorities, if any.

The first four entries in the notation can be filled with all elements mentioned in the list in Section 3.2. If any of the elements is not mentioned in these four fields, it is assumed to be fixed. If all unmentioned resource elements should default to stochastic instead, an $R$ can be written in the second field: the same goes for defaulting to controlled, dynamic or irrelevant elements. Analogously, a $D$ can be written in any of the first four fields to set a default for the demand elements. 
For the system characteristics, a notation is proposed that gives an answer to questions 1,2 and 3 of the identifiers. Thus: are there other authorities, is the information global or local and is optimisation global or local? The notation is based on Figure 1 [34]. In a similar way to this figure, the four options for the field system characteristics in the notation are:

- selfish: information global and optimisation local,

- social: information global and optimisation global,

- cooperative: information local and optimisation global,

- limited: information local and optimisation local.

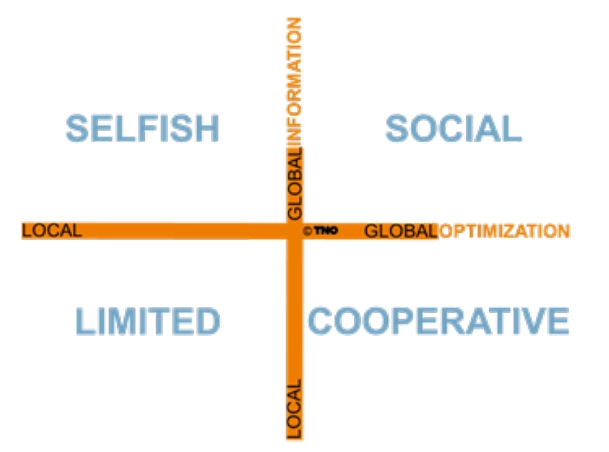

Fig. 1. Different models of a synchromodal network.

The four options for the final field are one turn only, equilibrium, isolated and 1: the first three are explained in Section 3.1, and the final option denotes that there are no other decision-making authorities in the system.

\subsection{Two-column notation}

Though the proposed six-field notation is a relatively compact way to describe a complex system, it comes with two downsides: it requires a degree of memorisation, and if new natures other than controlled, fixed, stochastic, dynamic or irrelevant are distinguished, there is no place for this in the current notation. These problems are solved by using the two-column notation described in this section, at the cost of compactness.

A synchromodal transportation model can also be described by the notation:

\begin{tabular}{|l|l|}
\hline Controlled elements & $C$, written out \\
\hline Fixed elements & fixed elements, written out \\
\hline Stochastic elements & $S$, written out \\
\hline Dynamic elements & $D$, written out \\
\hline Irrelevant elements & $I$, written out \\
\hline System characteristics & $Y$ \\
\hline Behaviour of other authorities & $B$ \\
\hline
\end{tabular}


If there are no stochastic elements in a problem, that row can be left out: the same goes for the other natures. If a new nature is distinguished, a row can be easily added for this. In the six-field notation, any unmentioned element was considered fixed, unless an $R$ or $D$ was placed in one of the fields to set the default to that nature. This is again possible here: an $R$ and a $D$ should always be placed in one of the rows to set the default nature of the resource elements and demand elements, respectively.

\subsection{On the two notations}

In neither notation, the optimisation objective is included: these are considered to be too distinct among different problems to merit classification. As discussed earlier, the two-column notation is much less compact than the six-field notation, but requires less memorisation and lends itself better to change when new natures are distinguished. Our advice is to employ the two-column notation at first, but to switch to the six-field notation when the framework starts gaining familiarity: this familiarity should make the memorisation easier, and this adoption time should suffice to discover any truly important new natures. This paper will largely use the six-field notation for the sake of compactness, seeing how reminders are readily available within this paper.

\section{$5 \quad$ Examples}

As discussed earlier, one of the ideas of the framework is that, when starting work on a new problem, one can first classify the assumptions this model would need, then investigate papers that have similar classification. Therefore, a number of classification examples are presented for both existing models and new problems. First, we answer the framework questions for the Kooiman pick-up case [21] in Table 2, and show how this can be written in our compressed notation. Afterwards, Table 3 shows compressed notation of some other problems described in papers, such that the interested reader can study more examples of our framework classification. Then, using Table 4, we examine some real-life cases and classify how we would choose to model these problems. To clarify: these problems do not yet have an explicitly described model, so this classification is based on how we would approach and model these practical problems, but other modellers may make other modelling decisions. Finally, the given examples will be used as input for discussion. In the Kooiman pick-up case [21], a barge makes a round trip along terminals in a fixed schedule to pick up containers to bring back to the main terminal; however, the arrival times of the containers at the terminals are stochastic. At each terminal, a decision has to be made of how many containers to load onto the barge, and a guess has to be made of how much capacity will be needed for later terminals, all while minimising the amount of late containers. The actual time of residing at the terminal is disregarded. We refer to Table 2 for the answering of the framework questions. We refer to Table 1 for a reminder of the framework element abbreviations. 
$R$ : unmentioned resource elements $\mid D$ : unmentioned demand elements

$R O$ : resource origin

$D O$ : demand origin

$R D$ : resource destination

$D D$ : demand destination

$R C$ : resource capacity

$D V$ : demand volume

$R D T$ : resource departure time

$R T T$ : resource travel time

$R P$ : resource price

$D R D$ : demand release date

$D D D$ : demand due date

$T H$ : terminal handling time

$D P$ : demand penalty

$D 2 R$ : demand-to-resource allocation

Table 1. Abbreviations of the framework elements used in the compressed notation.

\begin{tabular}{|l|l|}
\hline Other authorities & No \\
\hline Information global/local & Global \\
\hline Optimisation global/local & Global \\
\hline Resource elements & $\begin{array}{l}\text { Resource type: barges } \\
\text { Controlled resource elements: none } \\
\text { Resource features: fixed, except } T H \text { (irrelevant) }\end{array}$ \\
\hline Demand elements & $\begin{array}{l}\text { Demand type: freight containers } \\
\text { Controlled demand elements: } D 2 R \\
\text { Demand features: fixed, except } D R D \text { (stochastic) }\end{array}$ \\
\hline Optimisation objective & $\begin{array}{l}\text { Maximal percentage of containers that travel by } \\
\text { barge instead of truck }\end{array}$ \\
\hline
\end{tabular}

Table 2. The framework applied on the Kooiman pick-up case [21].

Note that only barges are taken into consideration as resources, not trucks. It would have been possible to describe trucks as resources as well, but we have chosen to classify these as part of the lateness penalty, because there is no decision-making in how the trucks are used. Also, it may seem strange to speak of global or local information and optimisation when there are no other decision-making authorities. The information is considered global, because the only decision-making authority knows 'everything' that happens in the network; the optimisation is considered global, because the decision-maker wants to optimise the performance over all demand in the network put together, not over some individual piece or pieces of freight.

Using the six-field notation, most of Table 2 can be summarised as follows:

$$
D 2 R|D R D| \cdot|T H| \text { social } \mid 1
$$

It could also be represented in the two-column notation, as follows:

\begin{tabular}{|l|l|}
\hline Controlled elements & Demand-to-resource allocation \\
\hline Fixed elements & $R, D$ \\
\hline Stochastic elements & Demand release date \\
\hline Irrelevant elements & Terminal handling time \\
\hline System characteristics & social \\
\hline Behaviour of other authorities & 1 \\
\hline
\end{tabular}

Here, the row for dynamic elements can be left out because the problem has no dynamic elements, and $R$ and $D$ are written in the row for fixed elements to indicate that any unmentioned resource element and any unmentioned demand element is fixed by default. 
Only the optimisation objective and type specifications are lost in this process. In Table 3, we apply the framework to more problems from academic papers. In this table, we include the optimisation objective to illustrate the wide range of optimisation possibilities. It is not actually necessary to describe the optimisation objective when using the compressed problem notation. In some cases, especially practical problem descriptions, optimisation objectives may not yet be explicitly known. Therefore, Table 4 leaves them out. In that table we review some practical problem descriptions and apply the framework to them.

Behdani [8]: D2R, RDT $|\cdot| \cdot|\cdot|$ social $\mid 1$

Objective: minimal transportation costs and waiting penalties

Kooiman [21]: D2R $|D R D| \cdot|T H|$ social $\mid 1$

Objective: maximal percentage of containers by barge instead of truck

Le Li [24]: $D 2 R|\cdot| D V|R D T, D R D, D D D|$ cooperative $\mid$ equilibrium

Objective: with self-optimising subnetworks, total minimal cost in union

Lin $[26]: D 2 R|\cdot| R C|R P|$ social $\mid 1$

Objective: minimal total quality loss of perishable goods

Mes [27]: $D 2 R|\cdot| R P|R C|$ social $\mid 1$

Objective: best modality paths against different balances of objectives

Nabais [30]: $D 2 R|\cdot| R C, R T T, R P, D V, D P|T H|$ social $\mid 1$

Objective: sustainable transport modality split that retains client satisfaction

van Riessen [37]: D2R, RDT $|\cdot| R C, R T T, R P, T H, D P|\cdot|$ social $\mid 1$

Objective: minimise transport and transfer cost, penalty for late delivery and cost of use of owned transportation

Rivera [31]: $D 2 R|D| R|\cdot|$ social $\mid 1$

Objective: minimal expected transportation costs

Theys [41]: RP,D2R,DP $|\cdot| \cdot|R D T, D R D, D D D|$ sel fish $\mid$ equilibrium

Objective: fairest allocation of individual costs

$\mathrm{Xu}[50]: D 2 R, R C|R P, D V, D P| \cdot|R D T, R T T, T H, D R D, D D D|$ social $\mid 1$

Objective: maximised expected profit during tactical planning

Zhang [51]: $D 2 R|D| \cdot|\cdot|$ social $\mid 1$

Objective: maximised balance of governmental goals

Table 3. Selected papers in the synchromodal framework.

\begin{tabular}{|l|}
\hline Lean and Green Synchromodal [1]: D2R $|\cdot| \cdot|\cdot|$ selfish $\mid 1$ \\
\hline Rotterdam - Moerdijk - Tilburg [3]: D2R|RTT,TH| $|\cdot|$ social $\mid 1$ \\
\hline Synchromodaily [4]: D2R, RDT $|D| \cdot|\cdot|$ social $\mid 1$ \\
\hline Synchromodal Control Tower [5]: D2R, RC, DV $|R P, R T T, T H| \cdot|\cdot|$ social $\mid 1$ \\
\hline Synchromodale Cool Port control [6]: D2R, RDT $|R T T| D D D, D P|\cdot|$ social $\mid 1$ \\
\hline
\end{tabular}

Table 4. Selected use cases in the synchromodal framework.

Another example we reviewed is the modelling of an agent-centric synchromodal network. Here all agents want to be at their destination as fast as possible, but everyone does share the information about where they are and where they are going with everybody else in the network. Table 5 shows the answer on the 
questions of the framework. In the short notation this problem is:

$$
D 2 R|D| \cdot|D P| \text { selfish } \mid \text { equilibrium }
$$

\begin{tabular}{|l|l|}
\hline Other authorities & Yes \\
\hline Information global/local & Global \\
\hline Optimisation global/local & Local \\
\hline Resource elements & $\begin{array}{l}\text { Resource type: barges, trains and trucks } \\
\text { Controlled resource elements: none } \\
\text { Resource features: fixed }\end{array}$ \\
\hline Demand elements & $\begin{array}{l}\text { Demand type: containers } \\
\text { Controlled demand elements: } D 2 R \\
\text { Demand features: stochastic, except } D P \text { (irrelevant) }\end{array}$ \\
\hline Optimisation objective & Minimise travel times \\
\hline
\end{tabular}

Table 5. The framework for an agent-centric synchromodal network.

\section{Solution method mapping}

In the previous section, a number of papers on synchromodal transport problems and solution methods were studied. Some of the choices in solution methods are similar between papers and can be partially recognised from their framework notation. Here, we group the papers on solution method with remarks on complexity issues and insightful framework similarities:

- Shortest path algorithms: In [27], D2R is to be performed under the absence of capacity constraints. Mes et al. rightfully note that, in the absence of capacity constraints, the best modality paths can be found simply by using shortest path algorithms, which are known to run in polynomial time in the input size. Whenever capacity is included, this brings computational difficulties, as dividing flow over capacitated arcs is related to the NP-hard multi-knapsack problem. In [51], this is handled by a sequential shortest path algorithm: whenever a demand item comes in, assign it to the cheapest path with remaining capacity and repeat this until everything is assigned. Though this, too, is an efficient method, one can imagine it yielding suboptimal results, especially under the stochastic release dates. However, if $D 2 R$ is the only control element, a sequential shortest path algorithm is a recognised as a computationally efficient option: in the absence of capacity constraints, stochastic elements and control-based dynamic elements, it is likely to yield the optimal solution.

- Two-stage stochastic programming: In [50], D2R must again be performed. $R C$ is technically a control element as well, but the challenge lies mainly in the $D 2 R$ control. Now, the stochasticity is dealt with by means of two-stage stochastic programming. The studied model may lend itself well to stochastic programming because no intermediary nodes are recognised between the one origin and the set of destinations. Even so, Xu et al. propose a meta-heuristic to deal with the computational intensity incurred by large sets of freight types, destinations, transportation modes or scenarios. 
- Approximate dynamic programming: In [21] and [31], Markov Decision Process models are presented but argued to be too computationally expensive. Instead, they solve $D 2 R$ with stochastic elements by making tentative decisions, simulating the potential results of this decision and their incurred costs, then taking the tentative decision with the lowest simulated expected cost. This is recognised as a computationally reasonable alternative to solving $D 2 R$ with stochastic elements.

- Systems and control theory: In [24], a cooperative $D 2 R$ equilibrium problem is studied rather than a social problem without other authorities. In [26] and [30], $D 2 R$ is performed while dynamic elements play an important role. Finding a good equilibrium with the other authorities, or settling on a good equilibrium between the control elements and the dynamic parameters that depend on control, is understandably modelled using systems and control theory. In two out of these three papers, Model Predictive Control is employed. However, the similarities between these three papers could also be explained by their shared authors.

- Multi-control integer linear programming: In both [8] and [37], not only D2R is controlled, but $R D T$ as well, as a form of partial resource schedule control. Both papers resort to using integer linear programs to find an optimal solution. As many of the variables in these programs are indexed on three sets, these methods are expected to scale poorly to larger instances. Efficient solution methods to problems where not only $D 2 R$ is controlled but the resource schedules as well, appears to be an open problem: though the Vehicle Routing Problem (VRP) comes to mind, Section 7 will address the challenges that synchromodality introduce to the VRP.

- Game theory: In [41], fair pricing must be determined in a system with selfish decision-makers. Understandably, steering this selfish behaviour is attempted by using game theory. Theys et al. note that the proposed techniques work for limited systems, but that moderately advanced synchromodal systems require advanced game-theoretical techniques.

One could put this the other way round and wonder, given a problem classification, what solution methods could be suitable and what complexity issues arise. To this, we give the following answer. Selfish problems have been investigated with game theory, but only moderately advanced synchromodal systems already seem to require advanced game theory. Cooperative problems have been studied using Model Predictive Control, for which commercial solvers exist. Social $D 2 R$ problems could be solved using sequential shortest path algorithms. These are efficient methods, but only optimal under the absence of capacity constraints, stochasticity and control-based dynamic elements. Under the presence of capacity constraints, $D 2 R$ problems are likely to be NP-hard due to their similarity to the multi-knapsack problem. To solve $D 2 R$ with stochastic elements, two-stage stochastic programming and Markov Decision Processes have been examined, but proposed to be computationally too expensive. Approximate Dynamic Programming and Xu's meta-heuristic are proposed as efficient alternatives. To solve $D 2 R$ with dynamic elements, Model Predictive Control and other systems and 
control theory techniques are proposed. To solve social $D 2 R$ and $R D T$ simultaneously, only large-scale integer linear programs have been proposed in the examined literature.

This is far from a complete mapping from framework classification to solution method. Components that are not described by the framework may be critical to the viability of a solution method, like the absence of intermediary locations in [50] facilitating two-stage stochastic programming. However, we believe that worthwhile relationships have been and can be drawn between framework classifications and potential solution methods.

\section{Relationship to VRP terminology}

When optimising the transport of freight using several vehicles, thus simultaneously determining $D 2 R$ and resource schedules, the Vehicle Routing Problem (VRP) immediately comes to mind. The VRP is a widely studied transport problem. In a sense, a framework for the classification of different VRP variants exists in the form of consensus: the Capacitated Vehicle Routing Problem (CVRP), the Vehicle Routing Problem with Pickup and Delivery (VRPPD), the Vehicle Routing Problem with Time Windows (VRPTW), subvariants and combinations of these variants are well-known and their definitions largely agreed upon [22, 25]. However, none of the papers investigated in Section 5 seem to involve themselves explicitly with VRP models. This can be explained and recognised by applying the developed framework on VRP variants.

The VRP, in its most classical sense, is the problem of minimising transport costs when dispatching $m$ vehicles from some depot node to service all other nodes exactly once. A synchromodal version of this is quite imaginable. The real-time flexibility aspect of synchromodality would mean that re-evaluations may occur where the vehicles 'start' at their current destination, but must still return to the depot, and the already visited nodes are taken out of the problem. The information sharing aspect of synchromodality can be assumed to already be part of the problem: the resources and demands can be assumed to be pooled from several parties and put under the control of a central operator. Under these minor assumptions, the synchromodal VRP lends itself to the following classification:

$$
D 2 R, R D|\cdot| \cdot|R C, R D T, R T T, T H, D V, D R D, D D D, D P| \text { social } \mid 1
$$

The decision-maker must simultaneously decide which service nodes are visited by which vehicle and in which order. Time and capacity constraints are not present and all related elements are irrelevant. Only the total 'price' of these routes is minimised: though this price may equal the travel time, the actual element of time does not influence the decision space, as long as release time, due times and time windows are absent. When adding vehicle capacities, the 
$R C$ and $D V$ become fixed rather than irrelevant, so the synchromodal CVRP is denoted by

$$
D 2 R, R D|\cdot| \cdot|R D T, R T T, T H, D R D, D D D, D P| \text { social } \mid 1
$$

When time windows are added, the $R D T$ becomes a control element and the $R T T, D R D, D D D, D P$ and sometimes the $T H$ becomes relevant. Note that soft and hard time windows are not necessarily classified differently: the demand penalty could be an arbitrarily high constant to simulate hard deadlines, but soft due dates may also come with fixed penalties that are not arbitrarily high. As such, the synchromodal Capacitated Vehicle Routing Problem with Time Windows (CVRPTW) could be classified as, depending on whether or not terminal handling times are observed,

$$
D 2 R, R D, R D T|\cdot| \cdot|T H| \text { social } \mid 1 \quad \text { or } \quad D 2 R, R D, R D T|\cdot| \cdot|\cdot| \operatorname{social} \mid 1
$$

If separate pickup and delivery locations are specified, this would still mean that each demand item has a fixed $D O$ and $D D$, so the Capacitated Vehicle Routing Problem with Time Windows and Pickup and Delivery (CVRPTWPD) would be classified the same way as the CVRPTW.

One of the most important differences between synchromodal VRP variants and the problems examined in Section 5 are laid bare by the framework notation: all synchromodal VRP variants have the resource destination as a control element, while none of the studied papers do. In fact, having the $R D$ as a control element is largely synonymous with having the responsibility of routing.

While this definitely helps in recognising the absence of vehicle routing in the studied papers, it does not yet explain it. The following explanations for the absence of vehicle routing in the studied papers are proposed:

- Papers with more control elements than just $D 2 R$ tend to resort to using large ILP's, making inclusion of the $R D$ as a control element computationally challenging;

- In many of the papers, the routes were already predetermined in a strategi$\mathrm{cal} /$ tactical phase, and only the day-by-day assignment remained as a problem on the operational level, possibly due to this computational intensity and the real-world implications of planning vehicles routes;

- Most Multiple Travelling Salesman Problem (mTSP)-based models, including most VRP variants, do not lend themselves to the concept of intermodality, thus synchromodality: while intermodal transport encourages that different vehicles take care of different parts of a container's journey, most mTSP-based models encourage that the entire voyage of one container is taken care of by one vehicle only [9].

We conclude that the class of synchromodal transport problems differs significantly from the classical VRP variants: as such, they require a classification scheme of their own. 


\section{Discussion}

The examples in Section 5 show some strengths and limitations of the classification framework, which are discussed in this section.

One of the goals of this framework was to offer guidance when tackling a new problem: as an example, if the problem from the Synchromodaily [4] case is modelled in a non-stochastic way, we can now see that it may be worthwhile to study the solution method presented by Behdani [8], because they then have a very similar compressed framework classification: in particular, the Synchromodaily case involves the same control elements. If such a record is kept of papers and models, this could greatly improve the efficiency of developments in synchromodal transport. This would fulfil the second goal of the framework: to collect literature on synchromodal transportation within a meaningful order.

The final goal of this framework was to expose and compare relationships between seemingly different problems: for example, we can now see that the problems described by Le Li [24] and Theys [41] have similarities, in that they investigate negotiation between parties and do not focus on timeliness of deliveries. Similarly, we can see that the model assumption Mes [27] makes in disregarding resource capacity, is an uncommon decision. In Section 6, it was argued that such similarities and dissimilarities can help explain the effectiveness of certain solution methods.

In the Synchromodaily case [4], our interpretation of the problem implies that the demand features are stochastic. However, the problem could also be approached in a deterministic way, depending on choices that the modeller and contractor make based on the scope of the problem, the requirements on the solution and the available information. This shows the most important limitation of the classification framework: what classification to assign to a problem or model remains dependent on modelling choices, as well as interpretation of problem descriptions. Even without the framework, however, modelling choices will always introduce subjective elements into how a real-world problem is solved. This framework can be used to consistently communicate these underlying model assumptions.

A second limitation of the framework is that, because of the large amount of elements described in it, two similar problems are relatively unlikely to fall in the exact same space in the framework because of their minor differences. Therefore, one should not only look for problems with the exact same classification, but also problems with a classification that is only slightly different. In a more general sense, solution methods may apply to far more than one of these very specific framework classes. If two problems have the exact same controlled elements, it is imaginable that their models and solution methodologies may largely apply to the other. As a point of future research, it could be interesting to further investigate which classification similarities are likely to imply solution similari- 
ties, which may also be a stepping stone towards a general solution methodology.

As a final limitation, the compressed notation does not reveal that the paper by Lin [26] and the 'Synchromodale Cool Port control' [6] case both focus on perishable goods. This shared focus is not only cosmetic: mathematically, it may imply objective functions and constraints not focused on in other cases. To combat this limitation, we advise anyone using the framework to offer both a compressed and an extended description of their problem or model.

\section{References}

1. Lean and green synchromodal. www.synchromodaliteit.nl/case/lean-and-greenbarge/, accessed: 2017-02-27

2. Ontwikkeling van een synchromodale planningstool. http://www.synchromodaliteit.nl/case/ontwikkeling-van-een-synchromodaleplanningstool/, accessed: 2017-01-04

3. Rotterdam - Moerdijk - Tilburg; een pilot met synchromodaal vervoer. www.synchromodaliteit.nl/case/rotterdam-moerdijk-tilburg-een-pilot-metsynchromodaal-vervoer/, accessed: 2017-02-27

4. Synchromodaily. www.synchromodaliteit.nl/case/synchromodaily/, accessed: 2017-02-27

5. Synchromodal control tower. www.synchromodaliteit.nl/case/synchromodalecontrol-tower/, accessed: 2017-02-27

6. Synchromodale cool port control. www.synchromodaliteit.nl/case/synchromodalecool-port-control/, accessed: 2017-02-27

7. Bandeira, D., Becker, J., Borenstein, D.: A DSS for integrated distribution of empty and full containers. Decision Support Systems 47(4), 383-397 (2009)

8. Behdani, B., Fan, Y., Wiegmans, B., Zuidwijk, R.: Multimodal schedule design for synchromodal freight transport systems. European Journal of Transport \& Infrastructure Research 16(3), 424-444 (2016)

9. Bektas, T.: The multiple traveling salesman problem: an overview of formulations and solution procedures. Omega 34(3), 209-219 (2006)

10. Bock, S.: Real-time control of freight forwarder transportation networks by integrating multimodal transport chains. European Journal of Operational Research 200(3), 733-746 (2010)

11. Caris, A., Macharis, C., Janssens, G.: Corridor network design in hinterland transportation systems. Flexible Services and Manufacturing Journal 24(3), 294-319 (2012)

12. Chen, L., Miller-Hooks, E.: Resilience: an indicator of recovery capability in intermodal freight transport. Transportation Science 46(1), 109-123 (2012)

13. Crainic, T.: Service network design in freight transportation. European Journal of Operational Research 122(2), 272-288 (2000)

14. Crainic, T., Gendreau, M., Dejax, P.: Dynamic and stochastic models for the allocation of empty containers. Operations Research 41(1), 102-126 (1993)

15. Di Francesco, M., Lai, M., Zuddas, P.: Maritime repositioning of empty containers under uncertain port disruptions. Computers \& Industrial Engineering 64(3), 827837 (2013) 
16. Erera, A., Morales, J., Savelsbergh, M.: Global intermodal tank container management for the chemical industry. Transportation Research Part E: Logistics and Transportation Review 41(6), 551-566 (2005)

17. Goel, A.: The value of in-transit visibility for supply chains with multiple modes of transport. International Journal of Logistics: Research and Applications 13(6), 475-492 (2010)

18. Graham, R., Lawler, E., Lenstra, J., Rinnooy Kan, A.: Optimization and approximation in deterministic sequencing and scheduling: a survey. Annals of Discrete Mathematics 5, 287-326 (1979)

19. Huang, M., Hu, X., Zhang, L.: A decision method for disruption management problems in intermodal freight transport. In: Intelligent Decision Technologies, pp. 13-21. Springer (2011)

20. Kendall, D.: Stochastic processes occurring in the theory of queues and their analysis by the method of the imbedded markov chain. The Annals of Mathematical Statistics pp. 338-354 (1953)

21. Kooiman, K., Phillipson, F., Sangers, A.: Planning inland container shipping: a stochastic assignment problem. 23rd International Conference on Analytical and Stochastic Modelling Techniques and Applications (2016)

22. Laporte, G.: The vehicle routing problem: An overview of exact and approximate algorithms. European Journal of Operational Research 59(3), 345-358 (1992)

23. Li, L.: Coordinated Model Predictive Control of Synchromodal Freight Transport Systems. Ph.D. thesis, Delft University of Technology TRAIL thesis series (2016)

24. Li, L., Negenborn, R.R., De Schutter, B.: Distributed model predictive control for cooperative synchromodal freight transport. Transport. Res. Part E (2016), http://www.sciencedirect.com/science/article/pii/S1366554515303069

25. Lin, C., Choy, K.L., Ho, G.T., Chung, S.H., Lam, H.: Survey of green vehicle routing problem: past and future trends. Expert Systems with Applications 41(4), 1118-1138 (2014)

26. Lin, X., Negenborn, R.R., Lodewijks, G.: Towards quality-aware control of perishable goods in synchromodal transport networks. IFAC-PapersOnLine 49(16), $132-137$ (2016)

27. Mes, M., Iacob, M.: Synchromodal transport planning at a logistics service provider. In: Logistics and Supply Chain Innovation, pp. 23-36. Springer (2016)

28. Miller-Hooks, E., Zhang, X., Faturechi, R.: Measuring and maximizing resilience of freight transportation networks. Computers \& Operations Research 39(7), 16331643 (2012)

29. Min, H.: International intermodal choices via chance-constrained goal programming. Transportation Research Part A: General 25(6), 351-362 (1991)

30. Nabais, J.L., Negenborn, R.R., Benitez, R.B.C., Botto, M.A.: A constrained MPC heuristic to achieve a desired transport modal split at intermodal hubs. In: Intelligent Transportation Systems-(ITSC), 2013 16th International IEEE Conference on. pp. 714-719. IEEE (2013)

31. Pérez Rivera, A., Mes, M.: Service and transfer selection for freights in a synchromodal network. Lecture Notes in Computer Science 9855, 227-242 (2016)

32. Pfoser, S., Treiblmaier, H., Schauer, O.: Critical success factors of synchromodality: Results from a case study and literature review. Transportation Research Procedia 14, 1463-1471 (2016)

33. Phillipson, F.: Creating timetables in case for disturbances in simulation of railroad traffic. In: Proceedings of the 45th International Conference on Computers \& Industrial Engineering (CIE45). pp. 1-8 (2015) 
34. Phillipson, F.: A thought on optimisation, complexity and self-organisation in synchromodal logistics. Tech. rep., TNO, The Netherlands (2017)

35. Pleszko, J.: Multi-variant configurations of supply chains in the context of synchromodal transport. LogForum 8(4) (2012)

36. Puettmann, C., Stadtler, H.: A collaborative planning approach for intermodal freight transportation. OR Spectrum 32(3), 809-830 (2010)

37. Riessen, B.V., Negenborn, R.R., Dekker, R., Lodewijks, G.: Service network design for an intermodal container network with flexible due dates/times and the possibility of using subcontracted transport. International Journal of Shipping and Transport Logistics 7(4), 457-478 (2015)

38. Song, D., Dong, J.: Cargo routing and empty container repositioning in multiple shipping service routes. Transportation Research Part B: Methodological 46(10), 1556-1575 (2012)

39. SteadieSeifi, M., Dellaert, N.P., Nuijten, W., Van Woensel, T., Raoufi, R.: Multimodal freight transportation planning: A literature review. European Journal of Operational Research 233(1), 1-15 (2014)

40. Tavasszy, L., Behdani, B., Konings, R.: Intermodality and synchromodality (2015)

41. Theys, C., Dullaert, W., Notteboom, T.: Analyzing cooperative networks in intermodal transportation: a game-theoretic approach. In: Nectar Logistics and Freight Cluster Meeting, Delft, The Netherlands. pp. 1-37 (2008)

42. Topaloglu, H.: A parallelizable dynamic fleet management model with random travel times. European Journal of Operational Research 175(2), 782-805 (2006)

43. Topaloglu, H.: A parallelizable and approximate dynamic programming-based dynamic fleet management model with random travel times and multiple vehicle types. In: Dynamic Fleet Management, pp. 65-93. Springer (2007)

44. Topaloglu, H., Powell, W.: A distributed decision-making structure for dynamic resource allocation using nonlinear functional approximations. Operations Research 53(2), 281-297 (2005)

45. Topaloglu, H., Powell, W.: Dynamic-programming approximations for stochastic time-staged integer multicommodity-flow problems. INFORMS Journal on Computing 18(1), 31-42 (2006)

46. Topaloglu, H., Powell, W.: Sensitivity analysis of a dynamic fleet management model using approximate dynamic programming. Operations Research 55(2), 319$331(2007)$

47. Van Binsbergen, A., Konings, R., Tavasszy, L., Van Duin, J.: Innovations in intermodal freight transport: lessons from europe. In: Papers of the 93th annual meeting of the Transportation Research Board, Washington (USA), Jan 12-16, 2014; revised paper. TRB (2014)

48. Van Riessen, B., Negenborn, R.R., Dekker, R.: Synchromodal container transportation: An overview of current topics and research opportunities. Computational Logistics 9335, 386-397 (2015)

49. Vinke, P.: Dynamic consolidation decisions in a synchromodal environment: Improving the synchromodal control tower. Master's thesis, University of Twente (2016)

50. Xu, Y., Cao, C., Jia, B., Zang, G.: Model and algorithm for container allocation problem with random freight demands in synchromodal transportation. Mathematical Problems in Engineering 2015 (2015)

51. Zhang, M., Pel, A.: Synchromodal hinterland freight transport: model study for the port of Rotterdam. Journal of Transport Geography 52, 1-10 (2016) 